\title{
Long-term Impacts of Energy Storage Providing Regulation on Power Plant Retirements and System Emissions
}

Jing Peng, Johanna L. Mathieu

Electrical Engineering \& Computer Science

University of Michigan

\{jingpeng, jlmath\}@umich.edu
Catherine Hausman

Ford School of Public Policy

University of Michigan

chausman@umich.edu

\begin{abstract}
Energy storage can provide a variety of economic and reliability benefits to the grid; however, the overall environmental impacts of storage are not always positive, as some studies have shown. In this paper, we explore the long-term impacts of using storage to provide frequency regulation. Specifically, using an optimization model that co-optimizes unit commitment, energy, and regulation capacity, we explore the effect of increasing penetrations of regulation-providing storage on dispatch, prices, profit, retirements, and long-term system-wide $\mathrm{CO}_{2}$ emissions. We also investigate how the impacts change when retired generators are replaced by renewables. We find that storage can lead to increases or decreases in emissions, depending on system parameters and whether renewables replace retired capacity. Additionally, we find that long-term impacts can be in different directions than short-term impacts. This points to the need for new mechanisms to ensure desired environmental outcomes are achieved when using so-called "green" technologies.
\end{abstract}

\section{Nomenclature}

$a_{g}, b_{g}, c_{g} \quad$ Quadratic heat rate curve coefficients of generator $g$

$B_{i j} \quad$ Susceptance of line $i j$

$E_{g} \quad \mathrm{CO}_{2}$ emissions rate of generator $g$

$f_{g} \quad$ Energy cost function of generator $g$

$k_{g}^{\mathrm{f}} \quad$ Fuel price of generator $g$

$k_{g}^{\mathrm{r}}, k_{s}^{\mathrm{r}} \quad$ Regulation capacity costs of generator $g$ and storage $s$

$k_{g}^{\mathrm{vm}}, k_{g}^{\mathrm{fm}} \quad$ Variable and fixed O\&M costs of generator $g$

This research was funded by the Alfred P. Sloan Foundation. $p_{g}$

$P_{g}^{\max }, P_{g}^{\min }$

$p_{i j}^{\mathrm{f}}$

$P_{i j}^{\mathrm{f}, \max }$

$P_{i}^{\text {load }}, P_{i}^{\text {load,s }}$

$r_{g}, r_{s}$

$R_{g}^{\max }, R_{s}^{\max }$

Agricultural \& Resource Economics

University of California, Berkeley

jesse_buchsbaum@berkeley.edu

Power generation of generator $g$

Maximum and minimum power generation limits of generator $g$

Power flow on line $i j$

Maximum power flow limit on line $i j$

Load and storage-induced load at bus $i$

Regulation capacity of generator $g$ and storage $s$

Maximum regulation capacity of generator $g$ and storage $s$

$R^{\text {req }} \quad$ Regulation requirement

$u_{g} \quad$ Unit commitment status of generator $g$

$y_{g} \quad$ Hourly operating profits of generator $g$

$\beta \quad$ Storage-induced load coefficient

$\theta_{i} \quad$ Voltage angle of bus $i$

$\theta_{i}^{\max }, \theta_{i}^{\min } \quad$ Maximum and minimum voltage angle limits of bus $i$

$\theta^{\text {ref }} \quad$ Voltage angle reference

$\pi^{\mathrm{p}}, \pi^{\mathrm{r}} \quad$ System-wide energy and regulation prices

\section{Introduction}

Technological improvements and a decline in cost have increased integration of energy storage into power systems. In particular, battery storage is highly flexible and able to provide high-value power systems services [1] such as frequency regulation, also known as secondary frequency control and henceforth referred to as simply regulation. The US Federal Energy Regulatory Commission (FERC) has also created 
favorable market conditions for storage. Since FERC Order 755 [2], which requires Independent System Operators (ISOs) to compensate regulation providers for both capacity and performance, large-scale battery storage capacity in the US has increased from $59 \mathrm{MW}$ in 2010 to more than $1 \mathrm{GW}$ in 2020 , with $17 \mathrm{GW}$ projected by 2050 [3]. As of 2018, about $75 \%$ of the capacity was used to provide regulation [3]. Furthermore, FERC Order 841 [4] removed most remaining barriers to storage participation in electricity markets, including ancillary services.

Energy storage providing regulation helps maintain system reliability, but it also has other impacts on the system. Importantly, it alters system dispatch of both energy and ancillary services, and it can also impact unit commitment $[5,6]$. This is because it replaces the sources that used to provide regulation, which in the US are usually a combination of natural gas, hydropower, and fast-ramping coal power plants [7]. ISOs in the US co-optimize energy and ancillary services and so changes in ancillary services bids directly affect the energy market. For example, a coal power plant that has traditionally provided regulation but is no longer dispatched to provide the service because of the entry of storage can generate at higher or lower levels because it does not have to reserve a margin from its minimum and maximum power generation limit to be able to provide regulation. This change affects generator profits in addition to system-wide costs and emissions. Although energy storage is often thought of as a "green" technology for its potential to help integrate more renewable energy, previous studies have found that storage providing regulation can decrease or increase system-wide $\mathrm{CO}_{2}$ emissions [6, 8]. However, these studies focused only on short-term impacts.

In this paper, we explore the long-term impacts of storage providing regulation considering short-term changes in dispatch, operational costs, and generator profits that in turn impact plant retirements and long-term emissions outcomes. Our goal is not to forecast emissions impacts for a particular system but instead to explore the range of outcomes that are physically possible given the way power systems are operated and the way generators make retirement decisions. More concretely, we wish to answer the question, "if emissions increase in the short term could they decrease in the long term?", and vice versa. To do this we apply an optimization model that co-optimizes unit commitment, energy, and regulation capacity to an IEEE test network, simulate the system over a 10-year period in which the capacity of storage providing regulation increases, and assess plant retirements and emissions outcomes.
A number of prior studies have explored the emissions impacts of storage. For example, [9] found that storage can reduce renewables curtailment and decrease $\mathrm{CO}_{2}$ emissions. In contrast, [10-13] found that energy storage providing arbitrage could increase emissions. None of these studies considered storage providing regulation. Ref. [14] considers storage providing multiple services including regulation but focuses on analyzing the overall value of storage rather than changes in emissions, and the results do not make clear the value/change-in-emissions due to storage providing regulation versus other services. Ref. [15] shows that vehicle-to-grid services provided by vehicle batteries could reduce emissions in Texas, but does not explore long-term impacts. Most similar to our work, [16] explores the long-term impacts of storage providing energy and/or ancillary services in Texas and finds long-term emissions will increase. In contrast to [16], which aims to forecast outcomes for a particular system, our paper aims to identify the range of possible outcomes and the reasons for those outcomes. For example, though [16] includes unit retirement in its planning model, the direct impacts of storage on retirements are not investigated, as they are here. Some papers study the impacts of storage through expansion planning models $[14,17,18]$ in which the goal is to make optimal investment decisions to achieve certain outcomes rather than to observe outcomes based on expected increases in storage penetrations, as we do here. Additionally, other papers have explored the impact of plant retirements on storage capacity [19] and value [20], and [21] studied how storage providing arbitrage affects the revenue of existing plants, but storage providing regulation was not modeled.

A key factor affecting long-term changes in emissions due to storage providing regulation is how the generation mix evolves following plant retirements. In the US, the Biden administration is championing a goal of electricity sector decarbonization by 2035 [22] achievable through timely retirement of fossil fuel capacity and replacement with renewable energy sources [23]. Therefore, in this paper we also explore how emissions outcomes differ when retired capacity is not immediately replaced versus when it is replaced by renewables. A number of papers have explored long-term electricity decarbonization strategies including storage and renewables [24-26], but these papers did not consider storage providing regulation.

The contributions of the paper are 1) to develop an approach to explore and link the short-term and long-term impacts of storage providing regulation on system-wide emissions considering plant retirements 
and possible replacement by renewables and 2) our findings that long-term emissions impacts could be positive or negative and, more importantly, that long-term impacts can be in different directions than short-term impacts. We also provide empirical evidence based on analysis of data from the PJM Interconnection that our results may be generalizable. These findings show that so-called "green" technologies like storage can have unintended consequences as they affect both short-term operation and the long-term generation mix. Further, our work points to the need for new mechanisms to ensure that long-term outcomes are in line with the goals of new technologies.

The rest of this paper is organized as follows: Section 2 presents our methods and Section 3 presents a case study; details of test system set-up are given in Section 3.1 and results are shown and discussed in Section 3.2. Section 4 summarizes our findings and provides ideas for future work.

\section{Methods}

In this section, we present our method to assess the long-term impacts of storage on retirements and emissions, which is summarized in Fig. 1. We first set the storage capacity and then solve the optimization model that co-optimizes unit commitment, energy, and regulation capacity four times, once for each season using different representative system-wide load levels. With these results, we compute the yearly operating profit of each generator and compare each generator's profit to its fixed operations and maintenance (O\&M) costs to determine whether the generator is unprofitable and should retire. Retirement decisions are only made at the end of each year, and once a plant retires it can no longer be committed. Following retirement, renewable energy sources could be added to the system to replace retired capacity, or not. We repeat this yearly analysis for 10 years, increasing the storage capacity each time, and finally assess the long-term emissions impacts. We next detail our assumptions, present the optimization model used to determine short-term impacts, and describe how we assess profits, make retirement decisions, compute emissions, and add renewable energy capacity.

\subsection{Assumptions}

We make a large number of simplifying assumptions so that we can focus on the impact of increasing storage penetrations alone on long-term emissions outcomes and also so that we can more easily interpret the results.

- To isolate the effects of increasing storage penetrations, we do not change load levels from year to year or allow fossil fuel generator expansion. For our parameterization of the test system, fossil fuel generator expansion is not required even when renewables are not added because there is sufficient generation capacity after all observed unit retirements.

- We use a single-period optimization model (extrapolating the results from a single hour to a whole season) and so ignore multi-period constraints like ramping limits and minimum up/downtime limits.

- We include the DC power flow equations in our optimization model to capture transmission line limits; however, we only present case study results for the uncongested system. Future work will explore the impact of congestion.

- We model only a single ancillary service product regulation - and assume provision of it is symmetric, i.e. a generator must provide the same amount of capacity in both the upward and downward directions.

- We assume storage provides only regulation. Although much work has focused on storage providing arbitrage, most storage in the US provides regulation [3].

- Generator costs captured in the model include fuel costs, variable O\&M costs, and regulation provision costs. Storage costs include regulation provision costs. We assume each generator incurs a fixed O\&M cost at the end of every year, modeled as constant over time.

- The model is a mixed-integer quadratic program (MIQP), and therefore nonconvex. For our profit analysis, we need energy and regulation prices, which, if the problem were convex, would be the Lagrange multipliers of certain constraints. However, Lagrange multipliers are not well-defined for MIQPs. Therefore, we solve the MIQP to obtain the optimal integer variables, fix them to their optimal values, and re-solve the resulting quadratic program $(\mathrm{QP})$ to obtain the prices.

- We assume the regulation signal is approximately energy neutral, i.e., resources are dispatched upwards as often as downwards. This means that storage starting the hour at $50 \%$ state of charge will end the hour at approximately $50 \%$ state of charge with the exception of the additional energy incurred due to inefficiency losses, which we include in the model as storage-induced load.

- We consider only $\mathrm{CO}_{2}$ emissions and assume emissions are a function of generator energy delivery only. The previous assumption implies that, for a particular power generation set-point, the act of providing regulation does not significantly change the amount of energy that a generator delivers. Also, while regulation provision will impact a generator's 


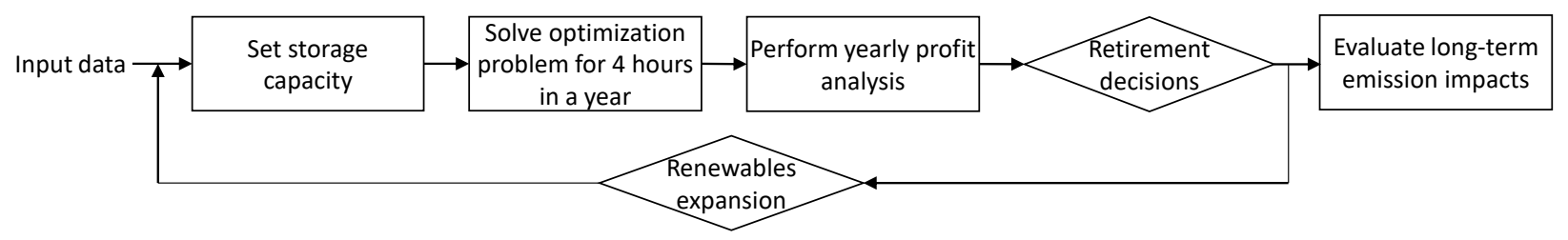

Figure 1. Flowchart of the method to assess the long-term impacts of storage on retirements and emissions.

heat rate and associated $\mathrm{CO}_{2}$ emissions, we assume this impact is small.

\subsection{Optimization model}

The optimization model co-optimizes unit commitment, energy, and regulation capacity as follows.

$$
\min \sum_{g, s} f_{g}\left(p_{g}, u_{g}\right)+k_{g}^{\mathrm{r}} r_{g}+k_{s}^{\mathrm{r}} r_{s}
$$

subject to

$$
\begin{aligned}
& f_{g}\left(p_{g}, u_{g}\right)=k_{g}^{\mathrm{f}}\left(a_{g} p_{g}^{2}+b_{g} p_{g}+c_{g} u_{g}\right)+k_{g}^{\mathrm{vm}} p_{g} \\
& \sum_{g \text { at bus } i} p_{g}-P_{i}^{\text {load }}-\sum_{s \text { at bus } i} P_{i}^{\mathrm{load}, \mathrm{s}}=\sum_{j} p_{i j}^{\mathrm{f}} \quad \forall i
\end{aligned}
$$

$$
\begin{aligned}
& p_{i j}^{\mathrm{f}}=B_{i j}\left(\theta_{i}-\theta_{j}\right) \quad \forall i j \\
& p_{g}+r_{g} \leq P_{g}^{\max } u_{g} \quad \forall g \\
& p_{g}-r_{g} \geq P_{g}^{\min } u_{g} \quad \forall g \\
& 0 \leq r_{g} \leq R_{g}^{\max } u_{g} \quad \forall g \\
& 0 \leq r_{s} \leq R_{s}^{\max } \quad \forall s \\
& \sum_{s} r_{s}+\sum_{g} r_{g} \geq R^{\text {req }} \\
& P_{i}^{\text {load,s }}=\sum_{s \text { at bus } i} \beta r_{s} \quad \forall i \\
& -P_{i j}^{\mathrm{f}, \max } \leq p_{i j}^{\mathrm{f}} \leq P_{i j}^{\mathrm{f}, \max } \quad \forall i j \\
& \theta_{i}^{\text {min }} \leq \theta_{i} \leq \theta_{i}^{\max } \quad \forall i \\
& \theta^{\text {ref }}=0 \\
& p_{g} \geq 0 \quad \forall g \\
& u_{g}: \text { binary } \quad \forall g
\end{aligned}
$$

where all notation is defined in the nomenclature section. The model is similar to that in [6], except [6] uses a multi-period version and assumes $c_{g}=0 \forall g$.
The decision variables are the power generation $p_{g}$ and commitment status $u_{g}$ of all generators $g$, regulation capacity $r_{g}, r_{s}$ of all generators $g$ and storage units $s$, power flows $p_{i j}^{\mathrm{f}}$ on all lines $i j$, and voltage angles $\theta_{i}$ at all buses $i$. The cost function (1) is the summation of the generation cost (2), generator regulation cost, and storage regulation cost, where the generation cost is the quadratic heat rate curve multiplied by the fuel price plus the variable $\mathrm{O} \& \mathrm{M} \operatorname{cost}^{1}$ and the regulation costs are assumed to be linear in the regulation capacity, and not functions of the power generation. Constraint (3) is the nodal power balance equation, (4) computes the power flows, (5) and (6) enforce generation limits, (7) and (8) enforce generator and storage regulation capacity limits, (9) is the regulation capacity requirement, (10) models the storage round-trip losses as an additional load as in [6] where $\beta$ is a parameter determined by the round-trip efficiency and regulation signal characteristics, (11) enforces power flow limits, (12) enforces voltage angle limits, (13) sets the voltage angle reference, (14) requires power generation to be non-negative, and (15) requires commitment to be binary.

\subsection{Profits, retirements, and emissions}

After solving the optimization problem, we calculate the profit of each generator using the dispatch results. As explained in Section 2.1, we use the Lagrange multipliers associated with (3) and (9) of the QP as the energy price $\pi^{\mathrm{P}}$ and regulation price $\pi^{\mathrm{r}}$, respectively. Then, the hourly operating profit of generator $g$ is

$$
y_{g}=\pi^{\mathrm{p}} p_{g}+\pi^{\mathrm{r}} r_{g}-f_{g}\left(p_{g}, u_{g}\right)-k_{g}^{\mathrm{r}} r_{g} .
$$

We multiply $y_{g}$ by the number of hours in a season $(2190 \mathrm{~h})$ to obtain the seasonal operating profit. Yearly operating profits are the summation of the four seasonal operating profits in a year. Yearly net profits are the difference between the yearly operating profits and the yearly fixed O\&M cost $k_{g}^{\mathrm{fm}}$.

If a generator's yearly net profit is negative, the generator may be retired. When multiple generators'

\footnotetext{
${ }^{1}$ The generation cost is zero when the generator is not committed. To enforce this, we multiply $c_{g}$ by $u_{g}$. Note that when $u_{g}=0$, $p_{g}=0$ and so the other terms will also be zero.
} 
profits turn negative in the same year, we allow only one retirement since retiring one generator increases the profitability of the others. At the end of the multi-year simulation, for each season across all 11 years, we calculate the hourly emissions of each generator by multiplying its heat energy (i.e., its heat rate curve evaluated at the dispatched $p_{g}$ ) by its emissions rate $E_{g}$, which is a function of its fuel type. Multiplying the hourly results by $2190 \mathrm{~h}$ and summing across the seasons in a year, we obtain the yearly emissions of each generator.

\subsection{Renewables expansion}

We explore two sets of cases, one in which retired capacity is not replaced and one in which all retired capacity is replaced by renewables. In the latter, a new renewable energy generator (e.g., a wind or solar farm) is added to the system after an existing generator is retired. We set its expected generation (i.e., its capacity times its capacity factor) equal to the retired capacity, and assume that it can be dispatched at levels less than or equal to its expected generation. We assume that the entry of renewables is realistic considering the overall reduction in wind and solar technology costs over the last decades [27], but we do not model renewable energy capital or installation costs because they do not influence the dispatch results. We assume that each renewable energy generator's marginal energy cost is zero and we allow it to provide regulation capacities up to $2 \%$ of its expected generation at a cost of $1 \$ / \mathrm{MW}$.

\section{Case Studies}

In this section, we present the results of a case study. We first introduce the system set-up and then detail the results of two cases with significantly different emissions outcomes.

\subsection{System Set-up}

We use the IEEE 9-bus system [28]; however, we change the line limits to $1000 \mathrm{MW}$ so that the system is always uncongested. We replace the existing generators with a total of 16 generators of different types: coal, natural gas combined cycle (CC), natural gas combustion turbine (CT), and oil, with parameters given in Tables 1 and 2 . We set the seasonal system loads to 500, 750, 600, and $700 \mathrm{MW}$. The system regulation requirement $R^{\text {req }}$ uses the $3+5$ rule, i.e., the required capacity is set equal to $3 \%$ of system load plus $5 \%$ of expected renewable generation [29]. Initially, there is no renewable generation in the system and so the regulation requirement is $3 \%$ of system load.
Table 1. Individual generator parameters

\begin{tabular}{lccccclc}
\hline Gen & Bus & $P^{\max } P^{\mathrm{min}}$ & $a$ & $b$ & $c$ & $k^{\mathrm{fm}}$ \\
& & MW & MW & $\frac{\text { MMBtu }}{\text { MW }^{2}}$ & $\frac{\text { MMBtu }}{\text { MW }}$ & MMBtu & $\frac{\$}{\text { MW-yr }}$ \\
\hline \hline Coal1 & 1 & 55 & 8 & 0.054 & 7.23 & 84.50 & 4500 \\
Coal2 & 1 & 55 & 17 & 0.025 & 8.50 & 70.35 & 4500 \\
Coal3 & 1 & 70 & 18 & 0.015 & 8.90 & 74.02 & 5000 \\
Coal4 & 2 & 70 & 16 & 0.036 & 7.55 & 55.21 & 5000 \\
Coal5 & 3 & 80 & 39 & 0.009 & 6.85 & 120.87 & 6000 \\
Coa16 & 2 & 90 & 38 & 0.000 & 8.40 & 75.98 & 7000 \\
CC1 & 2 & 45 & 19 & 0.076 & 4.15 & 88.30 & 800 \\
CC2 & 3 & 50 & 27 & 0.002 & 6.30 & 110.47 & 1000 \\
CC3 & 2 & 75 & 41 & 0.011 & 5.43 & 110.90 & 1500 \\
CT1 & 1 & 35 & 30 & 0.000 & 9.61 & -0.79 & 800 \\
CT2 & 1 & 35 & 29 & 0.000 & 9.59 & 1.35 & 800 \\
CT3 & 2 & 35 & 31 & 0.000 & 8.44 & 5.53 & 800 \\
CT4 & 3 & 40 & 12 & 0.000 & 10.70 & -13.64 & 800 \\
CT5 & 3 & 40 & 12 & 0.000 & 7.58 & 70.22 & 800 \\
CT6 & 2 & 55 & 25 & 0.000 & 9.86 & 2.01 & 800 \\
Oil & 2 & 20 & 15 & 0.000 & 5.87 & 72.06 & 0 \\
\hline
\end{tabular}

Table 2. Generator type-specific parameters

\begin{tabular}{lccccc}
\hline Type & $\begin{array}{c}k^{\mathrm{f}} \\
\frac{\$}{\mathrm{MMBtu}}\end{array}$ & $\begin{array}{c}R^{\max } \\
\% P^{\max }\end{array}$ & $\begin{array}{c}k^{\mathrm{r}} \\
\frac{\$}{\mathrm{MW}}\end{array}$ & $\begin{array}{c}k^{\mathrm{vm}} \\
\frac{\$}{\mathrm{MWh}}\end{array}$ & $\begin{array}{c}E \\
\frac{\text { ton }}{\mathrm{MMBtu}}\end{array}$ \\
\hline \hline Coal & 3 & 2 & 10 & 4 & 0.095 \\
CC & 4 & 5 & 6 & 2 & 0.053 \\
CT & 4 & 8 & 4 & 1 & 0.053 \\
Oil & 10 & 8 & 4 & 1 & 0.073 \\
\hline
\end{tabular}

Regulation costs are based on [7], O\&M costs on [30], and emissions factors on [31]. One storage system is added to Bus 7 with $k_{s}^{\mathrm{r}}=1 \$ / \mathrm{MW}$ and $\beta=0.038$ as in [6]. We run simulations for 11 years with $R_{s}^{\max }=0$ in year 0 and increasing by $5 \%$ of $R^{\text {req }}$ each year until reaching $50 \%$ of $R^{\text {req }}$ in year 10 .

Generator heat rate curves are estimated from 2013 hourly generation and heat-input data from the US CEMS database [32]. We picked a subset of generators to obtain a generation mix similar to that of the PJM Interconnection. We fit a quadratic curve to each generator's data between estimated minimum and maximum power generation limits, and we scaled down the generator capacities to approximately match those of the test system, using the scaling approach in [6].

\subsection{Results}

We present the results of two cases. In Case 1, we use the parameters in Table 1, while in Case 2, we change $c_{g}$ for CT5 to $60.22 \mathrm{MMBtu}$. In both cases, we find that the profits of both Coal1 and CT6 become negative at the end of year 5 . We explore 
what happens in each case if we 1) retire coal (Coal1) versus gas (CT6) without renewables expansion and 2) retire coal versus gas with renewables expansion, for a total of eight cases with retirements. We first detail illustrative results corresponding to Casel-retire coal. We then explore the range of possible results across all cases. Next, we seek to understand and generalize our results by analyzing the driving forces behind price changes, which affect profitability, retirements, and emissions outcomes. Finally, we present empirical results obtained from regression models fit with PJM data that characterize the relationship between prices and changes in the regulation requirement, which are used as a proxy for changes in storage capacity.

3.2.1. Illustrative results We first use Case1-retire coal as an example to explain how storage providing regulation affects generator dispatch, profitability, and emissions. Fig. 2 shows the optimal generation (left) and regulation (right) for coal (top), CC (middle), and CT (bottom) units. Horizontal lines show generation limits. In this case, the oil plant is never dispatched since it only needed on days with very high peak load, which are not modeled here. However, we do not retire it since we assume its fixed O\&M costs are extremely low. Each point on the plots shows the result for one season. Regulation from storage is always dispatched at its maximum because of its low cost.

In year 0 , there is no storage providing regulation. Although coal energy is the least expensive, coal has higher regulation costs and lower regulation capacities. Therefore, the system has to dispatch some expensive CT units to fulfill the regulation requirement, especially in low-load hours. For example, CT4 is operated near its minimum during all of year 0 , providing all of its regulation capacity. In year 2, with storage able to provide $10 \%$ of the regulation requirement, CT4 is de-committed and Coal2, Coal3, and Coal4 are operated at higher generation levels in season 1, reducing system costs. From year 2 to 5 , storage continues to provide more regulation and so units that produce less expensive energy (Coal6, CC2, and CC3) are able to generate at higher levels while other plants (Coal1, Coal4, and CT6) generate less and less resulting in a slight fuel switching from coal to gas. The addition of storage also leads to a gradual decrease in both energy and regulation prices. As a result, Coal1 and CT6 profit less and less and become unprofitable at the end of year 5. In this case, we retire Coal1, after which the energy it was providing is provided primarily by $\mathrm{CC}$ units operating at higher levels (CC1 and CT5) and secondarily by occasionally committing additional CT units (CT6).

Fig. 3 shows the yearly net profits of each generator.
The profits of all generators decrease from year 2 to 5 because of the drop in prices due to the addition of storage. At the end of year 5, Coal1 and CT6 both attain negative profit. Retiring Coal1 makes CT6 profitable. The opposite is also true (not shown). All generators benefit from Coal1's retirement as shown by the increase in profits from year 5 to 6 .

\subsubsection{Exploration of the range of possible results}

Fig. 4 shows the emissions outcomes for all four cases without renewables expansion in addition to benchmark cases with no retirements and with no storage. The dark red line with diamonds shows Case1-retire coal discussed in the previous subsection. Emissions increase in year 2 because of the generation shift from CT4 to Coal generators and then decrease gradually from year 2 to 5 because of the slight generation shift from coal to gas. After retiring Coal1, the emissions greatly decrease because of the shift from coal to gas. If instead of Coal1 retiring, CT6 is retired (Case1-retire gas, light red line with diamonds), emissions increase after year 5 because of a generation shift from gas to coal. In Case 2, emissions decrease before year 5 when, again, CT6 and Coal1 both become unprofitable. If we retire Coal1 (Case2-retire coal, dark blue line with diamonds) emissions continue to decrease, but if we retire CT6 (Case2-retire gas, light blue with diamonds) emissions significantly increase. For both cases, if units are not retired (dash-dot red and blue lines), the emissions results are between those of retiring coal and gas.

Fig. 5 shows the emissions outcomes for all four cases with renewables replacing retired capacity. From year 0 to 5 , the emissions outcomes are the same as in Fig. 4. Then emissions decrease significantly from year 5 to 10 due to the retirement of Coal1 or CT6 in year 5 and subsequent retirements in the following years, shown in Table 3. Both Cases 1 and 2 retire the same set of plants in the same order. In contrast to cases without renewables expansion, which retire only a single plant over 10 years, cases with renewables expansion retire six plants over 10 years. Because of its low marginal cost, renewables not only replace generation from retired capacity, but also some generation from operational and committed generators. This reduces fossil fuel generator profits, leading to further retirements and reductions in emissions.

Table 4 summarizes the emissions results for all eight cases showing average yearly emissions for year 0 (no storage), years 1 to 5 (pre-retirement), years 6 to 10 (post-retirement(s)), and all years 0 to 10 . In Case 1 without renewables expansion, emissions increase in the short-term, but can increase or decrease in the long-term 

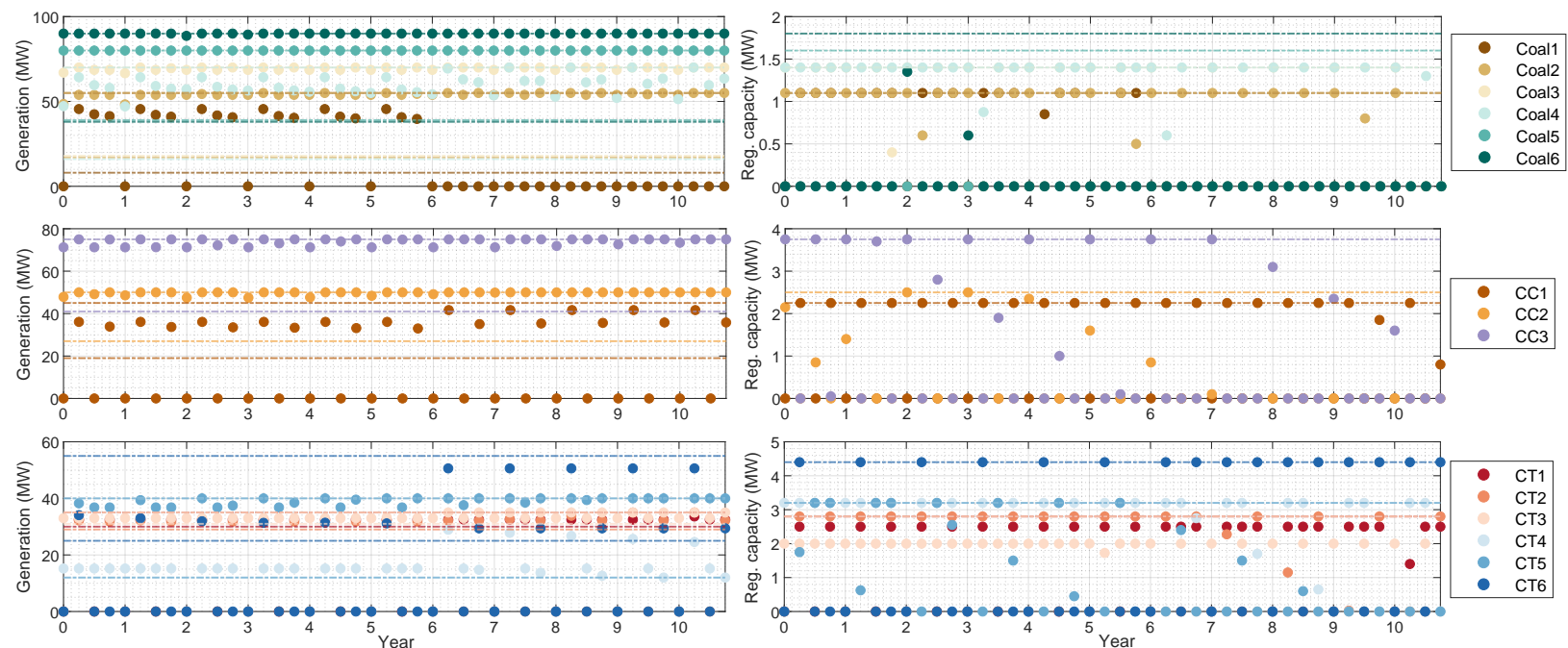

Figure 2. Optimal generation (left) and regulation (right) for coal (top), CC (middle), and CT (bottom) units for Case 1 when Coal1 retires in year 5 .

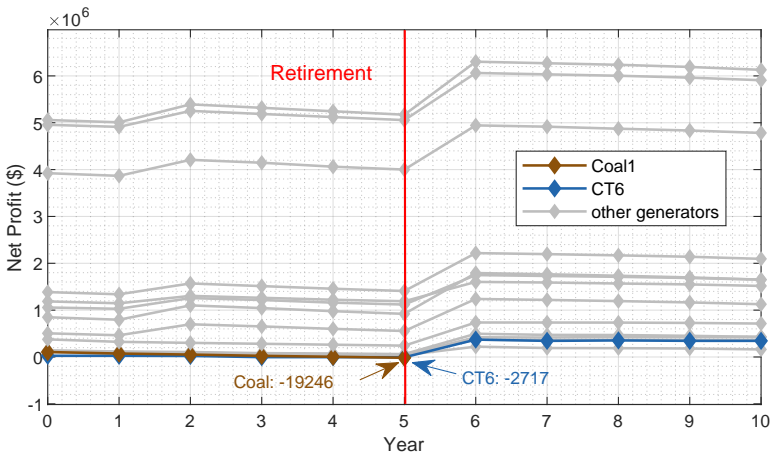

Figure 3. Yearly net profit for Case1 when Coal1 retires in year 5. CT6 and Coal1 both become unprofitable in year 5. Profitable generators are in grey.

Table 3. Retirements with renewables expansion

\begin{tabular}{lllllll}
\hline year & 5 & 6 & 7 & 8 & 9 & 10 \\
\hline \hline retire coal & Coal1 & CT6 & CC1 & CT2 & CT1 & Coal2 \\
retire gas & CT6 & Coal1 & CC1 & CT2 & CT1 & Coal2
\end{tabular}

depending upon which plant retires. In contrast in Case 2 , emissions decrease in the short-term but, similar to Case 1, can increase or decrease in the long-term. In both Cases 1 and 2 with renewables expansion, emissions decrease in the long-term.

3.2.3. Understanding price changes Generator profits are directly affected by energy and regulation prices, which are functions of the marginal energy and

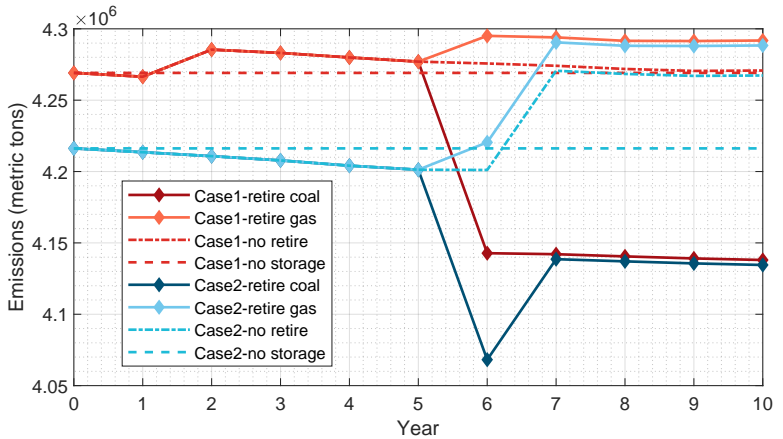

Figure 4. Yearly emissions for cases without renewables expansion.

regulation costs of generators on the margin. Fig. 6 shows yearly average energy prices and Fig. 7 shows yearly average regulation prices for all eight cases.

We use Case1-retire coal as an example to explain why energy and regulation prices change over time. In this case, energy prices decrease from year 0 to 1 , from years 2 to 5 , and from years 6 to 10 . During these time periods, storage replaces regulation capacity provided by both non-marginal generators (e.g., Coal2, $\mathrm{CC} 2$, and $\mathrm{CC} 3$ ) and marginal generators (e.g., Coal4 and $\mathrm{CC} 1$ ). As a result, non-marginal generators are able to produce more energy and marginal generators produce less energy, lowering energy prices. However, the energy price increases from year 1 to 2 . This is because storage growth causes changes in commitment status: CT4 is de-committed in year 2 season 1, as shown in Fig. 2 and described in Section 3.2.1. To compensate, marginal generators generate at higher 


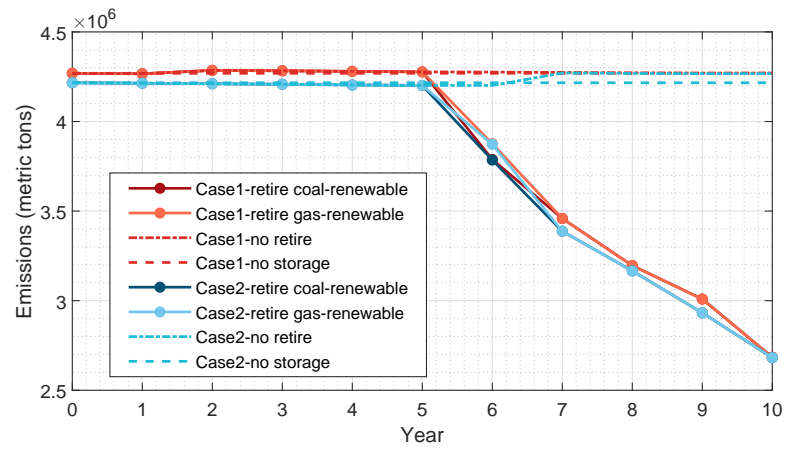

Figure 5. Yearly emissions for cases with renewables expansion.

Table 4. Average yearly emissions ( $10^{6}$ metric tons)

\begin{tabular}{lllll}
\hline & year & year & year & year \\
& 0 & $1-5$ & $6-10$ & $0-10$ \\
\hline \hline Case1-retire coal & 4.27 & 4.28 & 4.14 & 4.22 \\
Case1-retire gas & 4.27 & 4.28 & 4.29 & 4.28 \\
Case1-retire coal-renew. & 4.27 & 4.28 & 3.23 & 3.80 \\
Case1-retire gas-renew. & 4.27 & 4.28 & 3.25 & 3.81 \\
Case2-retire coal & 4.22 & 4.21 & 4.12 & 4.17 \\
Case2-retire gas & 4.22 & 4.21 & 4.28 & 4.24 \\
Case2-retire coal-renew. & 4.22 & 4.21 & 3.19 & 3.75 \\
Case2-retire gas-renew. & 4.22 & 4.21 & 3.21 & 3.75 \\
\hline
\end{tabular}

levels to meet the load, raising energy prices. Energy prices also increase from year 5 to 6 due to Coall's retirement, which leads to changes in commitment and the set of generators on the margin. Specifically, the remaining generators must produce more energy and more expensive generators become marginal (e.g., CT4). Similarly, in Cases1-retire gas, retirement of CT6 also causes changes in commitment and leads to increased energy prices. Case 2 show similar price trends to Case 1.

In contrast, in cases with renewables expansion, energy prices drop after the first retirement in year 5 because the system is not as constrained; the retired capacity has been replaced by low-cost renewables. Further retirements and increased renewables in subsequent years lead to energy price decreases in the long-term.

Regulation prices are affected by the marginal energy and regulation costs of the sets of generators on the margin for energy and regulation. More concretely, increasing system regulation capacity may require that the set of generators on the margin for regulation increase or decrease their energy production to move away from lower or upper operational limits to access that capacity. Then the set of generators on the margin for energy must respond by compensating the change in

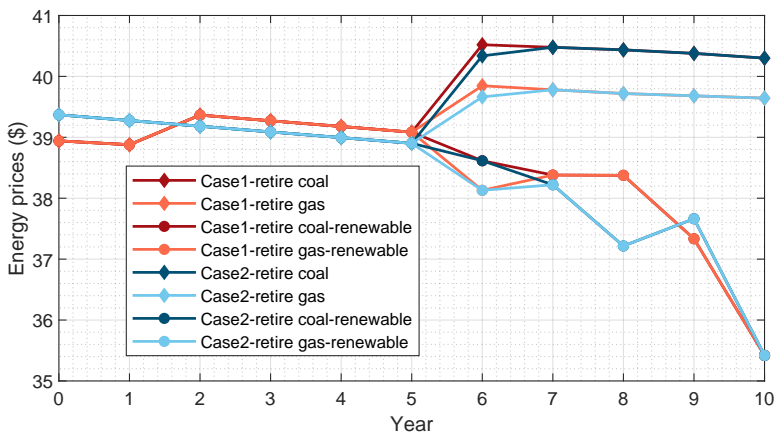

Figure 6. Yearly average energy prices.

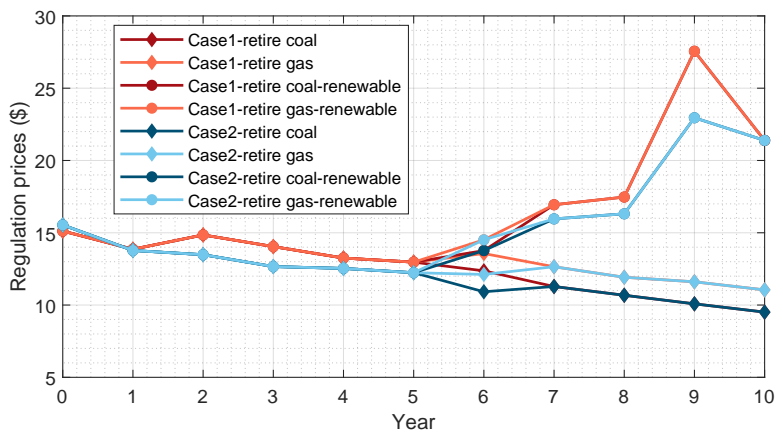

Figure 7. Yearly average regulation prices.

generation. Regulation prices are therefore the results of these combined changes in generator operation. In the long-term, without renewables expansion, regulation prices decrease because more and more storage provides inexpensive regulation. However, when renewables are added to the system, regulation prices increase in the long-term because the regulation requirement increases in accordance with $3+5$ rule.

3.2.4. Empirical results A key question is whether these results are specific to our case study, or generalizable. In our previous work focused on only short-term trends [8], we developed regression models to determine quantitative trends between regulation requirement changes and commitment, dispatch, and emissions outcomes in the short-term. We use this same method here to understand the relationship between storage capacity and prices. Specifically, we assume increased storage capacity causes an effective decrease in the regulation requirement, as storage capacity is generally used first for regulation, and determine the relationship between the regulation requirement and prices.

Using the same PJM data as we used in [8], we form a regression model $P_{t}=\alpha+\beta R_{t}+X_{t} \Theta+\varepsilon_{t}$, where $P_{t}$ is the market price, either for regulation or energy, 
Table 5. Empirically estimated changes in energy and regulation prices $(\$ / \mathrm{MWh})$ for a $100 \mathrm{MW}$ increase in storage capacity

\begin{tabular}{l|ccc}
\hline & OLS & Winsorized & Trimmed \\
\hline \hline Energy & $7.9^{*}$ & -0.8 & 0.4 \\
& $(4.0)$ & $(1.1)$ & $(0.5)$ \\
Regulation & 8.3 & -1.3 & $-2.1^{* *}$ \\
& $(6.2)$ & $(1.4)$ & $(0.8)$ \\
\hline
\end{tabular}

The unit of analysis is an hour. Values in parentheses are standard errors. ***, **,* indicate significance at the $1 \%$ and $5 \%$ and $10 \%$ level, respectively.

in hour $t ; R_{t}$ is the regulation requirement; $X_{t}$ is a vector of controls; and $\varepsilon_{t}$ is the residual. Standard errors are clustered by sample week. We control for load, retirements, quadratic time trend, fuel prices, weather, month, day of week, and hour effects; see [8] for details. We solve for the regression coefficients with ordinary least squares (OLS). To explore whether the presence of extreme price outliers drive our results, we rerun the regressions after winsorizing the top and bottom $5 \%$ of price observations and after trimming the top and bottom $5 \%$ of price observations. Our results are shown in Table 5.

Our empirical results show that when storage capacity increases, energy market prices increase (though the effect is small and disappears after trimming) and regulation market prices do not have a clear trend, but may decrease with increases in storage capacity. This is explained by a deeper analysis into the regression results in [8], which shows that when storage capacity increases, some less expensive coal units increase production to or near their capacity (and so they are not marginal) allowing some gas units to turn off. With this change in commitment and dispatch, gas or more expensive coal units may be marginal in the energy market, which increases energy market prices. Regulation market prices could increase or decrease, depending on which unit becomes marginal in the regulation market.

Again, taking Case1-retire coal as an example, the regression setting is most consistent with the change in year 1 to 2, when there are changes in commitment (as seen in the empirical results in [8]) and when there are no retirements (since the empirical results only capture short-term trends). Just like in the empirical results in Table 5, Fig. 6 shows an increase in energy prices due to the change in commitment caused by the increase in storage capacity. This finding provides some initial evidence that the qualitative trends we observe in our case study may be generalizable, but more work is needed. We acknowledge that the setting used for the regressions is not identical to that of our simulation study. Further, we might expect to see prices move in different directions in settings with different marginal units.

\section{Conclusions}

We have developed an approach to explore the short-term and long-term emissions impacts of storage providing regulation. Case study results show that storage providing regulation can decrease or increase emissions in both the short and long term. Moreover, long-term impacts are not necessarily consistent with short-term impacts, but may be. This finding is important for ISOs and policy makers since it highlights that so-called "green" technologies such as storage do not necessarily lead to improved environmental outcomes.

We also explored the impacts when retired generation capacity is replaced with renewables. In those cases, increases in renewable energy generally lead to decreases in energy prices and changes to dispatch that caused more fossil fuel unit retirements and significant decreases in emissions in the long-term.

Our future work includes developing more realistic case studies, exploring the design of mechanisms to achieve desired environmental outcomes, and conducting an empirical study of long-term emissions impacts, as we have done for short-term impacts in [8].

\section{References}

[1] P. Denholm, J. Jorgenson, M. Hummon, T. Jenkin, D. Palchak, B. Kirby, O. Ma, and M. O'Malley, "The value of energy storage for grid applications," Tech. Rep. TP-6A20-58465, NREL, 2013.

[2] FERC, "Order No. 755, Frequency regulation competition in organized wholesale power markets." https://www. ferc.gov/sites/default/f iles/2020-06/OrderNo.755.pdf, 2011.

[3] EIA, "Battery storage in the United States: An update on market trends." US Energy Information Agency, http s://www.eia.gov/analysis/studies/ele ctricity/batterystorage/pdf/battery_s torage.pdf, 2020.

[4] FERC, "Order No. 841, Electric storage participation in markets operated by regional transmission organizations and independent system operators." https : / / www . ferc.gov/media/order-no-841, 2018.

[5] Y. Lin, J. Johnson, and J. Mathieu, "Emissions impacts of using energy storage for power system reserves," Applied Energy, vol. 168, pp. 444-456, 2016.

[6] N. Ryan, Y. Lin, N. Mitchell-Ward, J. Mathieu, and J. Johnson, "Use-phase drives lithium ion battery life cycle environmental impacts when used for frequency regulation," Environmental Science \& Technology, vol. 52, no. 17, pp. 10163-10174, 2018.

[7] M. Hummon, P. Denholm, J. Jorgenson, D. Palchak, B. Kirby, and O. Ma, "Fundamental drivers of the 
cost and price of operating reserves," Tech. Rep. TP-6A20-58491, NREL, 2013.

[8] J. Buchsbaum, C. Hausman, J. Mathieu, and J. Peng, "Multi-product firms in electricity markets: Implications for climate policy," Working Paper 28027, National Bureau of Economic Research, 2020.

[9] M. Arbabzadeh, R. Sioshansi, J. X. Johnson, and G. A. Keoleian, "The role of energy storage in deep decarbonization of electricity production," Nature Communications, vol. 10, no. 1, pp. 1-11, 2019.

[10] E. S. Hittinger and I. M. Azevedo, "Bulk energy storage increases United States electricity system emissions," Environmental Science \& Technology, vol. 49, no. 5, pp. 3203-3210, 2015.

[11] R. Sioshansi, "Emissions impacts of wind and energy storage in a market environment," Environmental Science \& Technology, vol. 45, no. 24, pp. 10728-10735, 2011.

[12] R. Lueken and J. Apt, "The effects of bulk electricity storage on the PJM market," Energy Systems, vol. 5, no. 4, pp. 677-704, 2014.

[13] J. D. Ogland-Hand, J. M. Bielicki, Y. Wang, B. M. Adams, T. A. Buscheck, and M. O. Saar, "The value of bulk energy storage for reducing $\mathrm{CO} 2$ emissions and water requirements from regional electricity systems," Energy Conversion and Management, vol. 181, pp. 674-685, 2019.

[14] D. S. Mallapragada, N. A. Sepulveda, and J. D. Jenkins, "Long-run system value of battery energy storage in future grids with increasing wind and solar generation," Applied Energy, vol. 275, p. 115390, 2020.

[15] R. Sioshansi and P. Denholm, "Emissions impacts and benefits of plug-in hybrid electric vehicles and vehicle-to-grid services," Environmental Science \& Technology, vol. 43, no. 4, pp. 1199-1204, 2009.

[16] M. T. Craig, P. Jaramillo, and B.-M. Hodge, "Carbon dioxide emissions effects of grid-scale electricity storage in a decarbonizing power system," Environmental Research Letters, vol. 13, no. 1, p. 014004, 2018.

[17] M. Larsen and E. Sauma, "Economic and emission impacts of energy storage systems on power-system long-term expansion planning when considering multi-stage decision processes," Journal of Energy Storage, 2020.

[18] D. Liu, S. Zhang, H. Cheng, L. Liu, Z. Wang, D. Sang, and R. Zhu, "Accommodating uncertain wind power investment and coal-fired unit retirement by robust energy storage system planning," CSEE Journal of Power and Energy Systems, 2020.

[19] N. S. Pearre and L. G. Swan, "Renewable electricity and energy storage to permit retirement of coal-fired generators in Nova Scotia," Sustainable Energy Technologies and Assessments, vol. 1, pp. 44-53, 2013.

[20] P. Denholm, J. Jorgenson, M. Hummon, D. Palchak, B. Kirby, O. Ma, and M. O'Malley, "Impact of wind and solar on the value of energy storage," Tech. Rep. TP-6A20-60568, NREL, 2013.

[21] N. S. Goteti, E. Hittinger, B. Sergi, and I. L. Azevedo, "How does new energy storage affect the operation and revenue of existing generation?," Applied Energy, vol. 285, 2021.

[22] The White House, "Fact sheet: President Biden takes executive actions to tackle the climate crisis at home and abroad, create jobs, and restore scientific integrity across federal government." https://www. whitehouse .gov/briefing-room/statements-releas es/2021/01/27/fact-sheet-president-b iden-takes-executive-actions-to-tack le-the-climate-crisis-at-home-and-ab road-create-jobs-and-restore-scienti fic-integrity-across-federal-governm ent/, 2021.

[23] E. Grubert, "Fossil electricity retirement deadlines for a just transition," Science, vol. 370, 2020.

[24] N. Zhao and F. You, "Can renewable generation, energy storage and energy efficient technologies enable carbon neutral energy transition?," Applied Energy, vol. 279, 2020.

[25] D. B. Richardson and L. D. Harvey, "Optimizing renewable energy, demand response and energy storage to replace conventional fuels in Ontario, Canada," Energy, vol. 93, pp. 1447-1455, 2020.

[26] S. A. Maximov, G. P. Harrison, and D. Friedrich, "Long term impact of grid level energy storage on renewable energy penetration and emissions in the Chilean electric system," Energies, vol. 12, 2019.

[27] IRENA, "Renewable power generation costs in 2020." International Renewable Energy Agency, https : / / ww w.irena.org/-/media/Files/IRENA/Agen cy/Publication/2021/Jun/IRENA_Power_G eneration_Costs_2020.pdf, 2021.

[28] R. D. Zimmerman, C. E. Murillo-Sánchez, and R. J. Thomas, "MATPOWER: steady-state operations, planning, and analysis tools for power systems research and education," IEEE Transactions on Power Systems, vol. 26, no. 1, pp. 12-19, 2011.

[29] GE Energy, "Western wind and solar integration study," Tech. Rep. SR-550-47434, NREL, https : / / www.nr el.gov/grid/wwsis.html, 2010.

[30] EIA, "Generating unit annual capital and life extension costs analysis." US Energy Information Agency, http s://www.eia.gov/analysis/studies/pow erplants/generationcost/pdf/full_repor t.pdf, 2019.

[31] EIA, "Electric power annual." US Energy Information Administration, https://www.eia.gov/electr icity/annual/, 2019.

[32] EPA, "Continuous emission monitoring systems." US Environmental Protection Agency, https://www.ep a.gov/emc/emc-continuous-emission-mo nitoring-systems, Accessed 2019. 